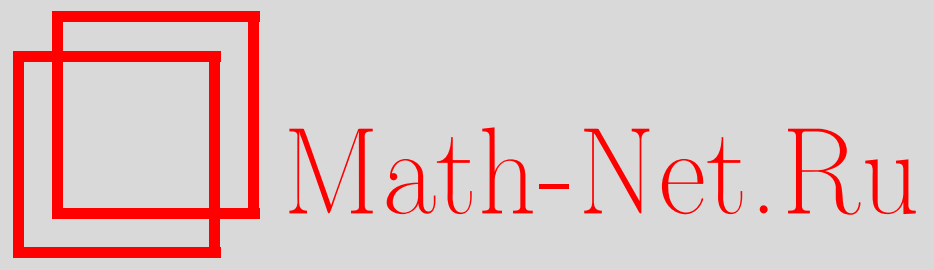

В. Ф. Гапошкин, Точные оценки метрической энтропии множества средних некоторых классов стационарных последовательностей, Теория вероятн. и ее примен., 2008, том 53, выпуск 1, 16-39

DOI: https://doi.org/10.4213/tvp317

Использование Общероссийского математического портала MathNet.Ru подразумевает, что вы прочитали и согласны с пользовательским соглашением

http: //www . mathnet.ru/rus/agreement

Параметры загрузки:

IP : 54.89 .56 .158

26 апреля 2023 г., 14:23:09

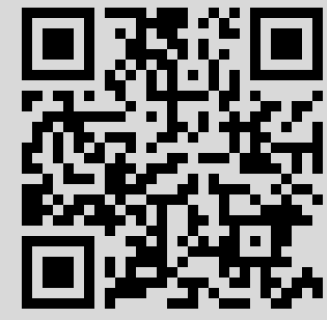


(C) 2008 г.

\author{
ГАПОШКИН В. Ф.*
}

\title{
ТОЧНЫЕ ОЦЕНКИ МЕТРИЧЕСКОЙ ЭНТРОПИИ МНОЖЕСТВА СРЕДНИХ НЕКОТОРЫХ КЛАССОВ СТАЦИОНАРНЫХ ПОСЛЕДОВАТЕЛЬНОСТЕЙ
}

Получены верхние и нижние оценки метрической $\varepsilon$-энтропии средних арифметических для классов стационарных последовательностей, совпадающие по порядку роста при $\varepsilon \rightarrow 0$. Эти оценки существенно отличаются от оценок для аналогичных классов квазистационарных последовательностей.

Ключевые слова и фразы: стационарные и квазистационарные последовательности, средние арифметические, метрическая энтропия, верхние и нижние оценки.

Пусть $\left(X_{k}\right)$ - стационарная последовательность в $L^{2}(\Omega), \mathbf{E} X_{k}=0$, $R_{1}(n)$ - ее корреляционная функция:

$$
R_{1}(n)=\mathbf{E} X_{k} X_{k+n}, \quad k=1,2, \ldots
$$

Если $A_{n}$ - средние арифметические последовательности $\left(X_{k}\right)$, т.е. $A_{n}=n^{-1} \sum_{k=1}^{n} X_{k}$, то обозначим

$$
\left\|A_{n}\right\|^{2}=n^{-2}\left\|\sum_{k=1}^{n} X_{k}\right\|^{2}=R(n) .
$$

Очевидно, что

$$
R(n)=n^{-1} R_{1}(0)+2 n^{-2} \sum_{k=1}^{n}(n-k) R_{1}(k) .
$$

Метрической энтропией $N(\varepsilon)$ множества $A=\left(A_{n}\right)_{1}^{\infty}$ называют наименьшее число замкнутых шаров радиуса $\varepsilon>0$ в $L^{2}(\Omega)$, покрывающих множество $A$. В работе [1] показано, что для любой стационарной последовательности справедлива оценка $N(\varepsilon)=O\left(\varepsilon^{-2}\right)$ при $\varepsilon \rightarrow 0$ и эта оценка неулучшаема в классе всех стационарных последовательностей. Но при добавочных ограничениях на спектральную плотность или корреляционную функцию последовательности оценки энтропии могут быть

* Московский государственный университет путей сообщения (МИИТ), ул. Образцова, 15, 101475 Москва, Россия; e-mail: gaposhkin@hotmail.com 
улучшены (см. [3]-[5]). В работах [4], [5] исследовались также квазистационарные последовательности. Последовательность $\left(X_{k}\right)$ называется $R$-системой, если $\left\|n^{-1} \sum_{k=m+1}^{m+n} X_{k}\right\|^{2} \leqslant R(n)(m \geqslant 0, n \geqslant 1)$, где $R(n)$ стремится к 0 . Всюду далее используется обозначение $\log n=\log _{2} n$.

Для $R$-систем в работе [5] получены следующие результаты:

1) если $\sum n^{-1}(R(n))^{1 / 2}<\infty$, то $N(\varepsilon)=O\left(\varepsilon^{-1}\right)$;

2) если $R(n)=O\left((\log n)^{-\alpha}\right)$, то $N(\varepsilon)=O\left(\varepsilon^{-1} \log \varepsilon^{-1}\right)$ (при $\left.\alpha=2\right)$ и $N(\varepsilon)=O\left(\varepsilon^{-2 / \alpha}\right)($ при $0<\alpha<2)$;

3) эти оценки неулучшаемы в соответствующих классах квазистационарных последовательностей.

В данной работе подробно исследуется более сложный случай стационарных последовательностей. Показано, что порядок роста энтропии $N(\varepsilon)$ при $\varepsilon \rightarrow 0$ существенно отличается от квазистационарного случая.

Удобно ввести следующие функции:

$$
F_{0}(n)=\sum_{k=1}^{n} R_{1}(k), \quad F_{1}(n)=\sum_{k=1}^{n} k R_{1}(k), \quad G(n)=n F_{0}(n)-F_{1}(n) .
$$

Тогда

$$
R(n)=n^{-1} R_{1}(0)+2 n^{-2} G(n) .
$$

Лемма 1. При $1 \leqslant n<n+l$

$$
\begin{aligned}
\left\|A_{n}-A_{n+l}\right\|^{2}=\frac{l^{2}}{(n+l)^{2}}( & \left(1+\frac{l}{n}\right)(R(l)-R(n+l)) \\
& \left.+\left(1+\frac{n}{l}\right)(R(n)-R(n+l))\right) .
\end{aligned}
$$

Д ок а 3 а т ел ь с т о. Имеем:

$$
A_{n}-A_{n+l}=\frac{l}{n+l}\left[n^{-1} \sum_{k=1}^{n} X_{k}-l^{-1} \sum_{j=n+1}^{n+l} X_{j}\right] \text {. }
$$

В силу стационарности

$$
\left\|A_{n}-A_{n+l}\right\|^{2}=\frac{l^{2}}{(n+l)^{2}}\left[R(n)+R(l)-\frac{2}{n l} D_{n, l}\right] .
$$

Здесь

$$
D_{n, l}=\sum_{k=1}^{n} \sum_{j=n+1}^{n+l} R_{1}(j-k) .
$$

Легко проверяется, что при $l<n$

$$
D_{n, l}=\sum_{k=1}^{l} k R_{1}(k)+l \sum_{k=l+1}^{n} R_{1}(k)+\sum_{k=n+1}^{n+l}(n+l-k) R_{1}(k) .
$$


Используя обозначения (1), получаем:

$D_{n, l}=F_{1}(l)+l\left(F_{0}(n)-F_{0}(l)\right)+(n+l)\left(F_{0}(n+l)-F_{0}(n)\right)-F_{1}(n+l)+F_{1}(n)$, что можно переписать в виде

$$
D_{n, l}=G(n+l)-G(n)-G(l)=\frac{1}{2}\left[(n+l)^{2} R(n+l)-n^{2} R(n)-l^{2} R(l)\right] .
$$

Подставляя последнее выражение в формулу (3), получим утверждение леммы при $l<n$. При $l \geqslant n$ выражение $D_{n, l}$ записывается в виде

$D_{n, l}=F_{1}(n)+n\left(F_{0}(l)-F_{0}(n)\right)+(n+l)\left(F_{0}(n+l)-F_{0}(l)\right)-F_{1}(n+l)+F_{1}(l)$, что приводит к тому же результату.

1. Верхние оценки энтропии средних. Из формулы (2) следует, что для точной оценки норм $\left\|A_{n}-A_{m}\right\|$ (как сверху, так и снизу) недостаточно налагать ограничения только на скорость убывания $R(n)$ (как в случае квазистационарных последовательностей), а нужны еще некоторые условия на поведение разностей $R(n)-R(m)$. Че́рез $C_{0}, C_{1}, D_{0}, D_{1}, \ldots$ в тексте обозначаются некоторые положительные постоянные, не обязательно одни и те же в разных неравенствах. Всюду далее предполагается, что $\lim _{n \rightarrow \infty} R(n)=0$.

Будем использовать следующее основное предположение.

Пусть существует такая неубываюшая функция $B(u), u \geqslant 1$, $\lim _{u \rightarrow \infty} B(u)=\infty$, что при некоторой постоянной $D_{1}>0$ имеем

$$
R(n)-R(m) \leqslant D_{1}\left(\frac{1}{B(\log n)}-\frac{1}{B(\log m)}\right) \quad(m>n \geqslant 2) .
$$

Заметим, что из (I) также следует оценка:

(Ia) $\quad R(n) \leqslant \frac{D_{1}}{B(\log n)}$.

3 а м е ч а н и е 1. Даже для квазистационарных последовательностей условие

$$
R(n)=O\left((\log n)^{-2-\delta}\right), \quad \delta>0,
$$

влечет «предельно малую» скорость роста $N(\varepsilon)=O\left(\varepsilon^{-1}\right)$ при $\varepsilon \rightarrow 0$. Поэтому нас, в основном, интересуют функции $B(u)$, удовлетворяющие условию

$$
B(u)=u^{\alpha}(\log u)^{\beta} \quad\left(u \geqslant u_{0}\right),
$$

где $\alpha>0, \beta \in \mathbf{R}$ или $\alpha=0, \beta>0$.

Основные верхние оценки при $\varepsilon \rightarrow 0$ энтропии множества средних $\left(A_{n}\right)$ содержатся в следующих теоремах. Пусть выполнены предположения (I) и (II). 
Теорема 1. 1) Если $\alpha>2$ или $\alpha=2, \beta>2$, mо

$$
N(\varepsilon)=O\left(\varepsilon^{-1}\right) \text {. }
$$

2) Если $\alpha=2, \beta=2, m o$

$$
N(\varepsilon)=O\left(\varepsilon^{-1} \log \log \log \varepsilon^{-1}\right) .
$$

3) Если $\alpha=2,-\infty<\beta<2$, mо

$$
N(\varepsilon)=O\left(\varepsilon^{-1}\left(\log \log \varepsilon^{-1}\right)^{(2-\beta) / 2}\right) .
$$

Теорема 2. Если $1<\alpha<2, \beta-$ любое, то

$$
N(\varepsilon)=O\left(\varepsilon^{-1}\left(\log \varepsilon^{-1}\right)^{(2-\alpha) / 2}\left(\log \log \varepsilon^{-1}\right)^{-\beta / 2}\right) .
$$

Теорема 3. Пусть $\alpha=1$. Тогда: 1) если $\beta>2$, то

$$
N(\varepsilon)=O\left(\varepsilon^{-1}\left(\log \varepsilon^{-1}\right)^{1 / 2}\left(\log \log \varepsilon^{-1}\right)^{1-\beta / 2}\right) ;
$$

2) если $\beta=2$, mo

$$
N(\varepsilon)=O\left(\varepsilon^{-1}\left(\log \varepsilon^{-1}\right)^{1 / 2}\left(\log \log \varepsilon^{-1}\right)\right) ;
$$

3) если $-\infty<\beta<2$, mо

$$
N(\varepsilon)=O\left(\varepsilon^{-1}\left(\log \varepsilon^{-1}\right)^{(3-\beta) / 2}\right) .
$$

Теорема 4. Если $0<\alpha<1, \beta-$ любое или $\alpha=0, \beta>0$, то

$$
N(\varepsilon)=O\left(\varepsilon^{-2 /(\alpha+1)}\left(\log \varepsilon^{-1}\right)^{-\beta /(\alpha+1)}\right) .
$$

Случай 1) теоремы 1 следует из цитированного выше результата для квазистационарных последовательностей. Действительно, в этом случае $\sum(R(n))^{1 / 2} n^{-1}<\infty$ и справедлива оценка $N(\varepsilon)=O\left(\varepsilon^{-1}\right)$. Впрочем, этот факт следует также из общей теоремы 5 . Для доказательства остальных результатов нужен ряд лемм. В некоторых из них удобно формулировать оценки энтропии для общих функций $B(u)$ из условия (I). При этом будет использоваться одно из условий

$$
\begin{aligned}
& \frac{B^{\prime}(u)}{B(u)} \leqslant \frac{C}{u}, \quad B^{\prime}(u) \text { не убывает при } u \geqslant u_{0}, \\
& B(u)=\frac{u}{\varphi(u)}, \quad \varphi(u) \text { не убывает при } u \geqslant u_{0} .
\end{aligned}
$$

Заметим, что если $B(u)$ имеет вид, указанный в условии (II), то выполнено условие (III) (при $\alpha>1$ или $\alpha=1, \beta \geqslant 0$ ), либо условие (IIIb) (при $0 \leqslant \alpha<1$ или $\alpha=1, \beta<0$ ).

Лемма 2. Пусть для стаиионарной последовательности $\left(X_{k}\right)$ выполнено условие (I). Пусть $m_{k}<m_{k+1}-$ некоторые челье числа, $3 m_{k} \geqslant m_{k+1}, l_{k}=m_{k+1}-m_{k} ;$ и пусть $m_{k} \leqslant n<m_{k+1}$. Обозначим для среднего $A_{n}$

$$
\rho_{k}(n)=\min \left(\left\|A_{n}-A_{m_{k}}\right\|,\left\|A_{n}-A_{m_{k+1}}\right\|\right) .
$$


Справедлива оченка $\rho_{k}^{2}(n) \leqslant D_{1} l_{k}^{2} m_{k}^{-2}\left(F_{k}+G_{k}\right)$, где

$$
\begin{aligned}
F_{k} & =\frac{1}{B\left(\log \left(l_{k} / 2\right)\right)}-\frac{1}{B\left(\log m_{k+1}\right)}, \\
G_{k} & =\frac{m_{k+1}}{l_{k}}\left(\frac{1}{B\left(\log m_{k}\right)}-\frac{1}{B\left(\log m_{k+1}\right)}\right) .
\end{aligned}
$$

Д о к а з а т е л ь с т в о. Очевидно, что либо $n-m_{k} \geqslant l_{k} / 2$, либо $m_{k+1}-n \geqslant l_{k} / 2$. Рассмотрим для определенности первый случай (второй разбирается вполне аналогично).

По лемме 1

$$
\begin{aligned}
\rho_{k}^{2}(n) \leqslant\left\|A_{n}-A_{m_{k}}\right\|^{2}=\frac{\left(n-m_{k}\right)^{2}}{n^{2}}( & \left(1+\frac{n-m_{k}}{m_{k}}\right)\left(R\left(n-m_{k}\right)-R(n)\right) \\
& \left.+\left(1+\frac{m_{k}}{n-m_{k}}\right)\left(R\left(m_{k}\right)-R(n)\right)\right) .
\end{aligned}
$$

Для завершения доказательства воспользуемся условием (I) и возрастанием функции $B(u)$, а также неравенствами

$$
\frac{l_{k}}{2} \leqslant n-m_{k} \leqslant l_{k}, \quad 1 \leqslant \frac{n}{m_{k}} \leqslant 3, \quad n<m_{k+1} .
$$

Лемма 3. Пусть выполнено условие (IIIa) или (IIIb). Тогда если $k, p$ - действительные числа, $k_{0} \leqslant k<k+p$ при некотором $k_{0} \geqslant 1, m o$

$$
\Delta=\frac{1}{B(k)}-\frac{1}{B(k+p)} \leqslant \frac{C p}{B(k)(k+p)} .
$$

Д о к а з а т е л ь с т в о. Действительно, при некотором $0<\theta<1$ имеем в случае (IIIa)

В случае (IIIb)

$$
\Delta=\frac{p B^{\prime}(k+\theta p)}{B(k) B(k+p)} \leqslant \frac{C p}{B(k)(k+p)} .
$$

$$
\Delta=\frac{\varphi(k)(k+p)-k \varphi(k+p)}{k(k+p)} \leqslant \frac{p}{B(k)(k+p)} .
$$

Следствие 1. Для функиий $B(u)$, удовлетворяюших условию (II), утверждение леммь 3 выполнено.

Д о к а з а т е л ь с т в о. Если $\alpha>1$ или $\alpha=1, \beta>0$, то функция

$$
B^{\prime}(u)=\alpha u^{\alpha-1}(\log u)^{\beta}\left(1+O\left((\log u)^{-1}\right)\right)
$$

возрастает при $u \geqslant u_{0}$; кроме того, при $u \geqslant u_{0}$

$$
B^{\prime}(u)(B(u))^{-1}=\alpha u^{-1}\left(1+O\left((\log u)^{-1}\right)\right) \leqslant C u^{-1} .
$$

В остальных случаях, очевидно, выполнено (IIIb). 
Лемма 4. Пусть $n, m, n<m$, - натуральные числа. Положим $n=2^{k}, m=2^{k+p}(k, p-$ действительные числа, $k \geqslant 0, p \geqslant 1)$. Если выполнены предположения (I) u (III), то при $k \geqslant k_{0}$ справедливы оченки:

$$
\begin{aligned}
R\left(2^{k}\right)-R\left(2^{k+p}\right) & \leqslant \frac{C_{2} p}{(k+p) B(k)}, \\
R\left(2^{k+p}-2^{k}\right)-R\left(2^{k+p}\right) & \leqslant \frac{C_{2} 2^{-p}}{(k+p) B(k)}, \\
\left\|A_{2^{k}}-A_{2^{k+p}}\right\|^{2} & \leqslant \frac{C_{2} p}{(k+p) B(k)} .
\end{aligned}
$$

Д о к а з а т е л ь с т в о. В силу (I) имеем

$$
\begin{aligned}
R\left(2^{k}\right)-R\left(2^{k+p}\right) & \leqslant D_{1}\left(\frac{1}{B(k)}-\frac{1}{B(k+p)}\right), \\
R\left(2^{k+p}-2^{k}\right)-R\left(2^{k+p}\right) & \leqslant D_{1}\left(\frac{1}{B\left(k+p-2^{-p+1}\right)}-\frac{1}{B(k+p)}\right) .
\end{aligned}
$$

Во втором неравенстве использованы также соотношения

$$
2^{k+p}-2^{k}=2^{k+p}\left(1-2^{-p}\right), \quad \log \left(2^{k+p}-2^{k}\right) \geqslant k+p-2^{-p+1} .
$$

Из леммы 3 следуют неравенства (5) и (6). Неравенство (7) получается из этих оценок и леммы 1.

Лемма 5а. Пусть выполнены условия (I) и (IIIа). Множество средних

$$
E_{2}=\left\{A_{n}: 2^{M} \leqslant n<2^{M^{2}}\right\}
$$

покрыцвается $\varepsilon_{2}$-сетью $S_{2}$ из не более чем $6 M$ элементов $A_{n_{1}}, \ldots, A_{n_{t}}$, $t \leqslant 6 M$. Здесь $\varepsilon_{2}=C_{2} / M, M \geqslant M_{0}$.

Д ок а з а т е л ь с т в о. Положим сначала для удобства $M=2^{\nu}$, $\nu \geqslant 1$. Образуем сеть $S_{2}$ из элементов

$$
n_{s}^{(j)}=2^{M 2^{j}+s 2^{2 j}}, \quad j=0, \ldots, \nu-1, \quad 0 \leqslant s \leqslant 2^{\nu-j} .
$$

Заметим, что $n_{0}^{(0)}=2^{M}$; максимальное из чисел $n_{s}^{(j)}$ равно $2^{M^{2}}$. Если $2^{M} \leqslant n<2^{M^{2}}$, то при некоторых $j, s$

$$
n_{s}^{(j)}=2^{M 2^{j}+s 2^{2 j}} \leqslant n<2^{M 2^{j}+(s+1) 2^{2 j}}=n_{s+1}^{(j)} .
$$

Пусть для определенности $n-n_{s}^{(j)} \geqslant n_{s+1}^{(j)}-n$. Положим в лемме 4

$$
k_{0}=M_{0}, \quad k=M 2^{j}+s 2^{2 j}, \quad k+p=\log n<M 2^{j}+(s+1) 2^{2 j} .
$$

Тогда $1 \leqslant p<2^{2 j}$. Так как $B^{\prime}(u)$ возрастает, то $B(u) \geqslant D_{3} u\left(u \geqslant u_{0}\right)$. В силу (7) имеем

$$
\left\|A_{n}-A_{n_{s}^{(j)}}\right\|^{2} \leqslant \frac{D_{4} 2^{2 j}}{M 2^{j} B\left(M 2^{j}\right)} \leqslant \frac{C_{2}^{2}}{M^{2}} .
$$


Всего элементов в сети $S_{2}$

$$
\operatorname{Card} S_{2} \leqslant \sum_{j=0}^{\nu-1}\left(2^{\nu-j}+1\right) \leqslant 3 \cdot 2^{\nu} .
$$

Если $M \geqslant M_{0}$ - произвольное, $2^{\nu-1}<M \leqslant 2^{\nu}$, то строим $C_{2} / 2^{\nu}$-сеть $S_{2}$ из не более чем $3 \cdot 2^{\nu}$ элементов. Она, очевидно, будет и $C_{2} / M$-сетью из не более чем $6 M$ элементов.

В лемме 6 исследуется базовый элемент конструкции $\varepsilon$-сетей, используемый далее в большинстве доказательств. В дальнейшем $] a[-$ наименьшее целое число, которое не меньше $a$.

Лемма 6. Пусть выполнены условия (I) $u$ (III). Пусть $r \geqslant 2-$ целое число, $M>8 ; H=H(r, M), H \geqslant 4$. а) Если $H>M$, положим

$$
\left.m_{0}=m_{0}^{(r)}=\right] M 2^{r}\left[, \quad m_{1}=m_{1}^{(r)}=\right] M 2^{r+1}[.
$$

б) Если $H \leqslant M$, то рассмотрим индексы

$$
\left.m_{k}=m_{k}^{(r)}=\right] M 2^{r+k H / M}\left[, \quad k=0, \ldots, K, \quad K=\left[\frac{M}{H}\right]+1 .\right.
$$

(При Фиксированном $r$ эти индексь не убььвают, $\left.m_{0}=\right] M 2^{r}\left[, m_{K}>\right.$ ] $M 2^{r+1}$ [.) Тогда при некотором $C>0$ для средних $A_{m_{k}}, A_{m_{k+1}}, A_{n}, m_{k}<$ $n<m_{k+1}$, справедлива оченка

$$
\rho_{k}^{2}(n) \leqslant C^{2} \frac{H^{2} \log (M / H+2)}{M^{2} B(r)(\log M+r)} .
$$

Д о к а 3 а т е л ь с т в о. В случае а) из леммы 2 легко следует, что для $m_{0}<n<m_{1}$

$$
\rho_{k}^{2}(n) \leqslant C^{2} \frac{1}{B(r)(\log M+r)},
$$

и оценка (9) подавно верна. В случае б) также используем лемму 2. Здесь $m_{k}=M 2^{r+k H / M}+\theta_{k}, m_{k+1}=M 2^{r+(k+1) H / M}+\theta_{k+1}, 0 \leqslant \theta_{k}, \theta_{k+1}<1$. Далее используются следующие неравенства:

$$
l_{k}=m_{k+1}-m_{k}, \quad \log \frac{l_{k}}{2} \geqslant r+\log H-2 \geqslant r, \quad 0<\frac{k H}{M}<1,
$$

$$
\begin{gathered}
\log M+r+\frac{(k+1) H}{M}+\frac{1}{M} \geqslant \log m_{k+1}, \quad \log M+r+\frac{k H}{M} \leqslant \log m_{k}, \\
\frac{H}{3 M} \leqslant \frac{l_{k}}{m_{k}} \leqslant 2 \frac{H}{M}, \quad \frac{m_{k+1}}{l_{k}} \leqslant \frac{6 M}{H} .
\end{gathered}
$$

Применяя леммы 2 и 3 , имеем

$$
\begin{aligned}
F_{k} & =\frac{1}{B\left(\log \left(l_{k} / 2\right)\right)}-\frac{1}{B\left(\log m_{k+1}\right)} \leqslant \frac{1}{B(r+\log H-2)}-\frac{1}{B(r+\log M+2)} \\
& \leqslant \frac{C_{1}(\log (M / H)+2)}{B(r)(r+\log M)},
\end{aligned}
$$




$$
\begin{aligned}
G_{k} & =\frac{m_{k+1}}{l_{k}}\left(\frac{1}{B\left(\log m_{k}\right)}-\frac{1}{B\left(\log m_{k+1}\right)}\right) \\
& \leqslant \frac{6 M}{H}\left(\frac{1}{B(\log M+r+k H / M)}-\frac{1}{B(\log M+r+(k+1) H / M+1 / M)}\right) \\
& \leqslant \frac{C_{2}}{B(r)(\log M+r)} .
\end{aligned}
$$

Поэтому при некоторой постоянной $C>0$ справедлива требуемая оценка леммы 6.

Для неубывающей функции $B(r)$ и $k \geqslant 2, M \geqslant 4$ обозначим

$$
\begin{aligned}
\Phi_{1}(k) & =\sum_{r=2}^{k}(B(r))^{-1 / 2}, \quad \Phi_{2}(k)=\sum_{r=2}^{k}(r B(r))^{-1 / 2} \\
\widetilde{\Phi}_{2}(M) & =\Phi_{2}([M])-\Phi_{2}([\log M]) \\
G(M) & =\Phi_{1}([\log M])+\sqrt{\log M} \widetilde{\Phi}_{2}(M) .
\end{aligned}
$$

Лемма 7а. Пусть выполнень условия (I) и (III). Пусть функиии $\Phi_{1}, \Phi_{2}, G$ определень согласно (10). Тогда существуют такие константы $D, C_{1}, D_{0}, M_{0}>0$, что при $M \geqslant M_{0}$ для множества средних $E_{1}=\left\{A_{n}: D_{0} M<n<2^{M}\right\}$ найдется $\varepsilon_{1}$-сеть $S_{1}\left(\right.$ с $\left.\varepsilon_{1}=C_{1} / M\right)$ такая, umo

$$
\operatorname{Card} S_{1} \leqslant D M G(M)
$$

Д оказатель с т в о. Выберем $r_{0} \geqslant 2$ так, чтобы при $r \geqslant r_{0}$ выполнялось неравенство $B(r) \geqslant 2^{4}$. Положим $D_{0}=2^{r_{0}}$. Составим нужную сеть $S_{1}$ из двух частей. При целых $r, r_{0} \leqslant r \leqslant \log M$, сеть $S_{1}^{\prime}$ состоит из $A_{n}$ с индексами $m_{k}^{(r)}$ вида $(8)$, где $H=H(r)=(B(r))^{1 / 2}$.

При $\log M<r \leqslant M$ сеть $S_{1}^{\prime \prime}$ состоит из $A_{n}$ с индексами вида (8), где

$$
H(r)=\left(r B(r)(\log M)^{-1}\right)^{-1 / 2} \text {. }
$$

Индексы $m_{k}^{(r)}$ не убывают с ростом $k$ и $r$; все они расположены между $] D_{0} M\left[\right.$ и $m_{K}^{(M)}>M 2^{M}$.

Пусть $A_{n}$ - некоторое среднее, $D_{0} M<n<2^{M}$. Фиксируем такие $k, r$, что

$$
m_{k}=m_{k}^{(r)} \leqslant n \leqslant m_{k+1}^{(r)}=m_{k+1}
$$

По лемме 6 при $M \geqslant M_{0}$

$$
\rho_{k}^{2}(n) \leqslant C_{1}^{2} \frac{H^{2}(r) \log (M / H+2)}{B(r)(r+\log M) M^{2}} .
$$

При $r_{0} \leqslant r \leqslant \log M$

$$
H^{2}(r)=B(r), \quad \log \left(\frac{M}{H}+2\right) \leqslant \log M \leqslant \log M+r,
$$


и правая часть в (12) не больше, чем $C_{1}^{2} / M^{2}$. При $\log M<r \leqslant M$ имеем

$$
H^{2}(r)=\frac{r B(r)}{\log M}, \quad \log \left(\frac{M}{H}+2\right) \leqslant \log M,
$$

и снова правая часть в (12) не превосходит $C_{1}^{2} / M^{2}$.

Составим при $M \geqslant M_{0}$ сеть $S_{1}$ из сети $S_{1}^{\prime}$ и из сети $S_{1}^{\prime \prime} ; \varepsilon_{1}=C_{1} / M$. Мы показали, что множество $S_{1}$ есть $\varepsilon_{1}$-сеть для $E_{1}$. Оценим $\operatorname{Card} S_{1}$. Имеем:

$$
\begin{aligned}
& \operatorname{Card} S_{1}^{\prime} \leqslant \sum_{r=2}^{[\log M]}\left(\frac{M}{H(r)}+2\right) \leqslant M \Phi_{1}([\log M])+2 \log M, \\
& \operatorname{Card} S_{1}^{\prime \prime} \leqslant M \sqrt{\log M}\left(\Phi_{2}([M])-\Phi_{2}([\log M])\right)+2 M .
\end{aligned}
$$

Отсюда следует оценка (11) при некоторой постоянной $D>0$.

Из лемм 5a, 7а вытекает следующая общая верхняя оценка энтропии.

Теорема 5. Пусть выполнень условия (I) и (IIIa), и пусть функиии $\Phi_{1}, \Phi_{2}, G$ определень согласно (10). Тогда при $M \geqslant M_{0}$ и некоторьх $C_{0}>0, \bar{D}>0$ для $\varepsilon=C_{0} / M$ справедлива оченка

$$
N(\varepsilon) \leqslant \bar{D} M G(M) .
$$

Д о к а з а т е л ь с т в о. Построим при $M \geqslant M_{0}$ сети $S_{2}, S_{1}$ по леммам 5а и 7а для множеств средних $E_{2}=\left\{A_{n}: 2^{M} \leqslant n<2^{M^{2}}\right\}, \varepsilon_{2}=C_{2} / M$, и $E_{1}=\left\{A_{n}: D_{0} M<n<2^{M}\right\}, \varepsilon_{1}=C_{1} / M$. Так как $B(u) \geqslant D_{3} u$, то при $n \geqslant 2^{M^{2}}$ в силу условия (Ia)

$$
\left\|A_{n}\right\|^{2} \leqslant R(n) \leqslant \frac{D_{1}}{B(\log n)} \leqslant \frac{C_{3}^{2}}{M^{2}},
$$

т.е. множество $E_{3}=\left\{A_{n}: n \geqslant 2^{M^{2}}\right\}$ содержится в шаре радиуса $C_{3} / M$ с центром в 0. Следовательно, множество

$$
S=S_{1} \cup S_{2} \cup\{0\} \cup\left(A_{1}, A_{2}, \ldots, A_{\left[D_{0} M\right]}\right)
$$

является $C_{0} / M$-сетью для множества $\left\{A_{n}: n \geqslant 1\right\}$, если положить $C_{0}=$ $\max \left(C_{1}, C_{2}, C_{3}\right)$. Мошность сети не больше, чем

$$
D M G(M)+D_{2} M \leqslant \bar{D} M G(M) \text {. }
$$

Доказательс т в о т ео рем 1,2 и 3 . Пусть $B(u)$ удовлетворяет условию (II). Если $\alpha>2$ или $\alpha=2, \beta>2$, то $G(M)=O(1)$. Это дает случай 1 ) теоремы 1 . Пусть теперь $1<\alpha \leqslant 2$. Согласно теореме 5 , нужно оценить функции $\Phi_{1}, \Phi_{2}, G$. Имеем:

$$
\Phi_{1}(k)=\sum_{r=2}^{k} r^{-\alpha / 2}(\log r)^{-\beta / 2}=O(\Psi(k)),
$$

где при $\alpha=\beta=2$, при $\alpha=2, \beta<2$ и при $1<\alpha<2$ и любом $\beta$ функция $\Psi(k)$ равна соответственно

$$
\log \log k, \quad(\log k)^{(2-\beta) / 2}, \quad k^{(2-\alpha) / 2}(\log k)^{-\beta / 2} .
$$


Так как при $\alpha>1$ ряд сходится, то $\widetilde{\Phi}_{2}(M) \leqslant \sum_{r=[\log M]}^{\infty} r^{-(1+\alpha) / 2}(\log r)^{-\beta / 2}=O\left((\log M)^{(1-\alpha) / 2}(\log \log M)^{-\beta / 2}\right)$.

Поэтому для функции $M G(M)$ получаются оценки теорем 1 и 2 . Теорему 5 можно применить и при $\alpha=1, \beta \geqslant 0$ (так как функция $B^{\prime}(u)$ не убывает). В этом случае

$$
\begin{aligned}
& \Phi_{1}(\log M)=O\left((\log M)^{1 / 2}(\log \log M)^{-\beta / 2}\right), \\
& \widetilde{\Phi}_{2}(M) \leqslant \sum_{r=[\log M]}^{M} r^{-1}(\log r)^{-\beta / 2}=O\left(\Psi_{1}(M)\right),
\end{aligned}
$$

где функция $\Psi_{1}(M)$ при $\beta>2, \beta=2, \beta<2$ равна

$$
(\log \log M)^{1-\beta / 2}, \quad \log \log M, \quad(\log M)^{1-\beta / 2}
$$

соответственно. Итак, порядок величины $G(M)$ определяется слагаемым $\sqrt{\log M} \widetilde{\Phi}_{2}(M)$. Отсюда получаются утверждения теоремы 3 (кроме случая $\alpha=1, \beta<0$, который исследуется далее).

Для доказательства оставшихся утверждений используем вместо лемм 5 а и 7 а приводимые ниже леммы 56 и 76.

Обозначим при $0<\alpha \leqslant 1$ или при $\alpha=0, \beta>0$ через $M_{1}$ корень уравнения $M_{1} B\left(M_{1}\right)=M^{2}$, а через $M_{2}$ - корень уравнения $B\left(M_{2}\right)=M^{2}$, $M \geqslant 2$. Очевидно, что при $\alpha>0$ и при $M \rightarrow \infty$

$$
M_{1} \sim C_{\alpha, \beta} M^{2 /(1+\alpha)}(\log M)^{-\beta /(1+\alpha)}, \quad M_{2} \sim D_{\alpha, \beta} M^{2 / \alpha}(\log M)^{-\beta / \alpha} .
$$

Если же $\alpha=0, \beta>0$, то

$$
M_{1} \sim \bar{M}_{1}=\frac{M^{2}}{\left(\log M^{2}\right)^{\beta}}, \quad \bar{M}_{1}<M_{1}, \quad M_{2}=2^{M^{2 / \beta}} .
$$

Лемма 5б. Пусть выполнень предположения (I), (II), где $0<\alpha \leqslant 1$ или $\alpha=0, \beta>0$. Тогда для множества средних $F_{2}=$ $\left\{A_{n}: 2^{M_{1}} \leqslant n \leqslant 2^{M_{2}}\right\}$ сушествует $\varepsilon_{2}$-сеть, где $\varepsilon_{2}=C_{2} / M$, причем

$$
\text { Card } S_{2}=O\left(M_{1}\right) \quad(M \rightarrow \infty) .
$$

Д о к а з а т е л ь с т в о. Пусть сначала $\alpha>0$ и $\beta$ - любое. Положим

$$
\begin{gathered}
n_{s}^{(j)}=\left[2^{M_{1} 2^{j}+s 2^{j(\alpha+1)}}\right], \\
\left.s=0,1, \ldots, s_{j}, \quad j=0,1, \ldots, J, \quad s_{j}=\right] \frac{M_{1}}{2^{j \alpha}}\left[, \quad J=\left[\log \frac{M_{2}}{M_{1}}\right] \sim \frac{\log M_{1}}{\alpha} .\right.
\end{gathered}
$$

Составим сеть $S_{2}$ из средних $A_{n}$ с этими номерами. Заметим, что наименьший из них равен $\left[2^{M_{1}}\right]$, а наибольший превосходит $2^{M_{2}}$. Далее,

$$
\operatorname{Card} S_{2} \leqslant \sum_{j=0}^{J}\left(\frac{M_{1}}{2^{j \alpha}}+1\right) \leqslant M_{1} C_{\alpha}+J+1=O\left(M_{1}\right) \text {. }
$$


Пусть $n_{s}^{(j)} \leqslant n \leqslant n_{s+1}^{(j)}$. Считая для определенности, что $n-n_{s}^{(j)} \geqslant$ $n_{s+1}^{(j)}-n$, обозначим в лемме $4 n_{s}^{(j)}=2^{k}, n=2^{k+p}$. Тогда при $M \geqslant M_{0}$ имеем

$$
\left\|A_{n}-A_{n_{s}^{(j)}}\right\|^{2} \leqslant \frac{C_{0} p}{B(k)(k+p)} \leqslant \frac{C_{1}^{2}}{M_{1}^{1+\alpha}\left(\log M_{1}\right)^{\beta}} \leqslant \frac{C_{2}^{2}}{M^{2}} .
$$

Здесь использованы неравенства

$$
\begin{gathered}
1 \leqslant p \leqslant 2^{j(\alpha+1)}, \quad B(k) \geqslant B\left(M_{1} 2^{j}\right), \quad k+p \geqslant M_{1} 2^{j}, \\
C_{3}\left(\log M_{1}\right)^{\beta} \leqslant \log ^{\beta}\left(M_{1} 2^{j}\right)=\left(\log M_{1}+j\right)^{\beta} \leqslant C_{4}\left(\log M_{1}\right)^{\beta} .
\end{gathered}
$$

Рассмотрим случай $\alpha=0, \beta>0$. Положим при $M \geqslant M_{0}$

$$
\left.k_{M}=\right] \frac{M^{2}}{\log ^{\beta}\left(M^{2} / \log ^{\beta} M\right)}[\text {. }
$$

Пусть при $k=1, \ldots, k_{M}$

$$
\left.m_{k}=\right] 2^{n_{k}}\left[, \quad n_{k}=2^{p_{k}}, \quad p_{k}=\left(\frac{M^{2}}{k}\right)^{1 / \beta} .\right.
$$

Числа $m_{k}$ подобраны так, чтобы $\left(\log \log m_{k}\right)^{-\beta} \sim k M^{-2}$. Покажем, что $\left(A_{1}, \ldots, A_{m_{k_{M}}}\right)$ есть $\varepsilon_{2}$-сеть $S_{2}$ для $F_{2}=\left\{A_{n}: 2^{M_{1}}<n<2^{M_{2}}\right\}$ при некотором $C_{2}>0$ и $\varepsilon_{2}=C_{2} / M, M_{1}, M_{2}$ удовлетворяют (15). При этом $\operatorname{Card} S_{2}=k_{M}=O\left(M_{1}\right)$. Заметим, что здесь удобна нумерация

$$
m_{1}>m_{2}>\cdots>m_{k_{M}}, \quad m_{1} \geqslant\left[2^{M_{2}}\right], \quad m_{k_{M}}=\left[2^{M_{1}}\right] .
$$

Для $m_{k}<n<m_{k-1}, l_{k}=m_{k-1}-m_{k}$, снова для определенности, считаем, что $n-m_{k} \geqslant l_{k} / 2$, и оценим по лемме 1

$$
\begin{aligned}
\| A_{n} & -A_{m_{k}} \|^{2}=\left(\frac{n-m_{k}}{n}\right)^{2} \\
& \times\left[\frac{n}{m_{k}}\left(R\left(n-m_{k}\right)-R(n)\right)+\frac{n}{n-m_{k}}\left(R\left(m_{k}\right)-R(n)\right)\right] .
\end{aligned}
$$

Легко проверяется, что $1 \leqslant m_{k-1} / l_{k} \leqslant 2$. По условию (I)

$$
\begin{aligned}
\frac{n}{n-m_{k}}\left(R\left(m_{k}\right)-R(n)\right) & \leqslant D_{1} \frac{m_{k-1}}{l_{k}}\left(B^{-1}\left(\log m_{k}\right)-B^{-1}\left(\log m_{k-1}\right)\right) \\
& =O\left(M^{-2}\right),
\end{aligned}
$$

так как

$$
\left(\log \log m_{k}\right)^{-\beta}-\left(\log \log m_{k-1}\right)^{-\beta}=O\left(\frac{k}{M^{2}}-\frac{k-1}{M^{2}}\right)=O\left(M^{-2}\right) .
$$

Остается оценить первое слагаемое в правой части $\left(15^{\prime}\right)$. Имеем:

$$
\frac{n}{n-m_{k}} \leqslant 4, \quad \log \frac{l_{k}}{2} \sim \log m_{k-1} \sim n_{k-1}, \quad \log \log \frac{l_{k}}{2} \sim p_{k-1} .
$$


По условию (I) и теореме Лагранжа при $1 \leqslant k \leqslant k_{M}$ и некоторых $0<\theta_{k}<1, u_{k}=n-m_{k} \theta_{k}$ имеем

$$
\begin{aligned}
& \frac{n}{m_{k}}\left(R\left(n-m_{k}\right)-R(n)\right) \\
& \quad \leqslant D_{1} \frac{n}{m_{k}}\left(\left(\log \log \left(n-m_{k}\right)\right)^{-\beta}-(\log \log n)^{-\beta}\right) \\
& \quad \leqslant \frac{D_{2} n}{u_{k} \log u_{k}\left(\log \log u_{k}\right)^{\beta+1}}=O\left(n_{k-1}^{-1} p_{k-1}^{-\beta-1}\right)=O\left(M^{-2}(\log M)^{-1}\right) .
\end{aligned}
$$

Последнее равенство следует из оценки: при $M \geqslant M_{0}$

$$
p_{k-1}^{\beta+1} n_{k-1} \geqslant p_{k_{M}}^{\beta+1} n_{k_{M}} \geqslant C_{\beta}\left(\log \frac{M^{2}}{\log ^{\beta} M}\right)^{\beta+1} \frac{M^{2}}{\log ^{\beta} M} \geqslant d_{\beta} M^{2} \log M .
$$

Поэтому из $\left(15^{\prime}\right)$ при некоторой постоянной $C_{2}>0$ получается нужная оценка:

$$
\left\|A_{n}-A_{m_{k}}\right\| \leqslant \frac{C_{2}}{M}, \quad k \leqslant k_{M}, \quad m_{k}<n<m_{k-1} .
$$

Лемма 7б. Пусть выполнены условия (I) и (II), где $0<\alpha \leqslant 1$, $\beta$-любое или $\alpha=0, \beta>0$. Рассмотрим множество средних

$$
F_{1}=\left\{A_{n}: 4 M \leqslant n \leqslant 2^{M_{1}}\right\} .
$$

Cуществует $\varepsilon_{1}$-сеть $S_{1}$ для $F_{1}, \varepsilon_{1}=C_{1} / M$ u Card $S_{1}=O\left(L_{M}\right)$. При $\alpha=1, \beta<2$ или $0 \leqslant \alpha<1$ функция $L_{M}$ равна соответственно

$$
M(\log M)^{(3-\beta) / 2}, \quad M^{2 /(1+\alpha)}(\log M)^{-\beta /(1+\alpha)} .
$$

Доказ а тель с т в о. Пусть $\left.2 \leqslant r \leqslant r_{M}, r_{M}=\right] M_{1}-\log M[$, $M \geqslant M_{0}$, и пусть $H=H(r)$ определено по формуле

$$
H(r)=B_{1}(r)\left(\log \frac{M}{B_{1}(r)}+1\right)^{-1 / 2}+4, \quad B_{1}(r)=(r B(r))^{1 / 2} .
$$

Рассмотрим индексы $m_{k}^{(r)}$, образованные по формулам (8) с этой функцией $H(r)$. Множество этих индексов расположено между ]4M[ и ]$M 2^{r_{M}+1}\left[>2^{M_{1}}\right.$. Так как $(\log x+1)^{1 / 2} \leqslant x$ при $x \geqslant 1$, то

$$
\begin{gathered}
\frac{M}{B_{1}} \geqslant 1, \quad \frac{M}{H} \leqslant \frac{M}{B_{1}}\left(\log \frac{M}{B_{1}}+1\right)^{1 / 2} \leqslant\left(\frac{M}{B_{1}}\right)^{2}, \\
\log \left(\frac{M}{H}+2\right) \leqslant 4\left(\log \frac{M}{B_{1}}+1\right) .
\end{gathered}
$$

По лемме 6 , используя обозначения (4), имеем

$$
\rho_{k}^{2}(n) \leqslant \frac{C^{2}}{M^{2}} A(r)
$$

где

$$
A(r)=\frac{r B(r) \log (M / H+2)}{(r+\log M) B(r)\left(\log \left(M / B_{1}\right)+1\right)} \leqslant 4 .
$$


Итак, для множества $F_{1}$ построена $\varepsilon_{1}$-сеть с $\varepsilon_{1}=C_{1} / M, C_{1}=2 C$. При этом

$$
\operatorname{Card} S_{1} \leqslant M \Sigma_{M}+2 r_{M}
$$

Здесь

$$
\Sigma_{M}=\sum_{r=2}^{r_{M}} r^{-(1+\alpha) / 2}(\log r)^{-\beta / 2}\left(\log \frac{M}{B_{1}(r)}+1\right)^{1 / 2} .
$$

Если $\alpha=1, \beta<2$, то воспользуемся оценками

$$
\log \frac{M}{B_{1}(r)}+1=O(\log M), \quad r_{M}=O\left(M(\log M)^{-\beta / 2}\right)
$$

и получим

$$
\Sigma_{M}=O\left((\log M)^{(3-\beta) / 2}\right), \quad \text { Card } S_{1}=O\left(M(\log M)^{(3-\beta) / 2}\right) .
$$

Если же $0<\alpha<1$, то нужна более точная оценка суммы $\Sigma_{M}$. Положим для удобства $\gamma=(1+\alpha) / 2,] \log M_{1}\left[=\nu\right.$. Тогда $M_{1} \leqslant 2^{\nu}$, $r_{M} \leqslant 2^{\nu}$, и в силу (14) при $\nu \rightarrow \infty$

$$
M \sim D_{\alpha, \beta} M_{1}^{\gamma}\left(\log M_{1}\right)^{\beta / 2}, \quad \log M \sim \gamma \nu+\frac{\beta \log \nu}{2}, \quad B_{1}(r)=r^{\gamma}(\log r)^{\beta / 2} .
$$

Представим сумму $\Sigma_{M}$ в виде

$$
\begin{aligned}
\Sigma_{M} & =\sum_{r=2}^{r_{M}} r^{-\gamma}(\log r)^{-\beta / 2}\left(\log M-\log B_{1}(r)+1\right)^{1 / 2} \leqslant \sum_{j=1}^{\nu} \sum_{r=2^{j-1}+1}^{2^{j}} \cdots \\
& \leqslant D_{1} 2^{\nu(1-\gamma)} \sum_{j=1}^{\nu} 2^{-(\nu-j)(1-\gamma)} j^{-\beta / 2}(\nu-j+1)^{1 / 2}=D_{1} \Sigma_{M}^{\prime} .
\end{aligned}
$$

Здесь использована оценка при $2^{j-1}<r \leqslant 2^{j}$ :

$$
\left(\log M-\log B_{1}(r)+1\right)^{1 / 2} \leqslant D_{2}(\nu-j+1)^{1 / 2} .
$$

Сумма $\Sigma_{M}^{\prime}$ оценивается так:

$$
\Sigma_{M}^{\prime} \leqslant 2^{\nu(1-\gamma)}\left(\sum_{j \leqslant \nu / 2}+\sum_{j>\nu / 2}\right) \leqslant D_{3} 2^{\nu(1-\gamma)} \nu^{-\beta / 2}=O\left(M_{1}^{1-\gamma}\left(\log M_{1}\right)^{-\beta / 2}\right) .
$$

Теперь из (17) получается нужная оценка Card $S_{1}$.

Доказательство те оремы 4 . Пусть $M \geqslant 2$. Выберем $M_{1}, M_{2}$ согласно (14), (15). При $n \geqslant 2^{M_{2}}$ имеем по условию (Ia)

$$
\left\|A_{n}\right\|^{2} \leqslant \frac{D_{1}}{B(\log n)} \leqslant \frac{D_{1}}{B\left(M_{2}\right)} \leqslant \frac{C_{3}^{2}}{M^{2}},
$$

т.е. множество средних $F_{3}=\left\{A_{n}: n \geqslant 2^{M_{2}}\right\}$ содержится в шаре радиуса $\varepsilon_{3}=C_{3} / M$ с центром в 0 . Далее, по лемме 56 для множества $F_{2}=\left\{A_{n}: 2^{M_{1}}<n<2^{M_{2}}\right\}$ построим сеть $S_{2}$ с $\varepsilon_{2}=C_{2} / M$, Card $S_{2}=O\left(M_{1}\right)$, а по лемме 76 для множества $F_{1}=\left\{A_{n}: 4 M \leqslant n \leqslant 2^{M_{1}}\right\}$ 
построим сеть $S_{1}$ с $\varepsilon_{1}=C_{1} / M$, Card $S_{1}=O\left(M_{1}\right)$. Положим $\varepsilon=C_{0} / M$, $C_{0}=\max \left\{C_{1}, C_{2}, C_{3}\right\}$. Очевидно, что тогда все множество средних $\left\{A_{n}: n \geqslant 1\right\}$ содержится в объединении $k_{M}$ шаров радиуса $\varepsilon$ с центрами в элементах сетей $S_{1}$ и $S_{2}$, а также в элементах $0, A_{1}, \ldots, A_{[4 M]}$. При этом

$$
k_{M}=O\left(M^{2 /(1+\alpha)}(\log M)^{-\beta /(1+\alpha)}\right) .
$$

Подставляя $M=C_{0} / \varepsilon$, получим оценку энтропии при $\varepsilon \rightarrow 0$

$$
N(\varepsilon)=O\left(\varepsilon^{-2 /(1+\alpha)}\left(\log \varepsilon^{-1}\right)^{-\beta /(1+\alpha}\right) .
$$

С помощью лемм 56 и $7 б$ разбирается и оставшийся в теореме 3 случай $\alpha=1, \beta<0$. Повторяя рассуждения из доказательства теоремы 4 , строим $C_{0} / M$-сеть, в соответствии с (14) здесь $M_{1} \sim C_{\beta} M(\log M)^{-\beta / 2}$, $M_{2} \sim D_{\beta} M^{2}(\log M)^{-\beta / 2}$. По леммам 56 и 76 мощность сети не превосходит

$$
D_{0} M+D_{1} M(\log M)^{(3-\beta) / 2}+D_{2} M(\log M)^{-\beta / 2}=O\left(M(\log M)^{(3-\beta) / 2}\right) .
$$

2. Нижние оценки энтропии средних. При оценках энтропии $N(\varepsilon)$ снизу естественно наложить некоторые «нижние» ограничения на функцию $R(n)$. Основным будет следующее условие.

Существует неубывающая функция $B(u)$ при $u \geqslant 1$ такая, что $\lim _{u \rightarrow \infty} B(u)=\infty$ и при $m>n \geqslant 2$ справедлива оценка

$$
R(n)-R(m) \geqslant D_{2}\left(\frac{1}{B(\log n)}-\frac{1}{B(\log m)}\right) .
$$

Хотя некоторые нижние оценки можно получать также при «общих» функциях $B(u)$ (в терминах $\Phi_{1}, \Phi_{2}, G$, как в теореме 5), мы ограничимся более конкретным случаем, когда для $B(u)$ выполнено условие (II). Покажем, что в этой логарифмической шкале все оценки предыдущего пункта являются точными по порядку. Получим сначала несколько вспомогательных утверждений. Следующая лемма очевидна и хорошо известна.

Лемма 8. Пусть $K$ - некоторое множество в нормированном пространстве $X$ и $\varepsilon>0$. Если в $X$ существуют такие элементы $x_{1}, \ldots, x_{l}$, ито $\left\|x_{i}-x_{j}\right\|>2 \varepsilon(i \neq j)$, то энтропия $N(K, \varepsilon) \geqslant l$.

Следующая лемма будет нужна для оценки расстояния между $A_{n}$ и $A_{m}$ при большой разнице номеров $n, m$.

Лемма 9. Пусть выполнены условия (I') и (II) для стачионарной последовательности $\left(X_{k}\right)$. Тогда при $1 \leqslant n<m, 2 n \leqslant m, \delta>\alpha+1$, $m \geqslant m_{0}$ справедлива оценка

$$
\left\|A_{n}-A_{m}\right\|^{2} \geqslant \frac{D_{\delta}}{(\log m)^{\delta}} .
$$


Д о к а з а т е л ь с т в о. По лемме 1 и свойству $\left(\mathrm{I}^{\prime}\right)$ имеем

$$
\left\|A_{n}-A_{m}\right\|^{2} \geqslant D^{2} \frac{(m-n)^{2}}{m^{2}}\left(B^{-1}(\log n)-B^{-1}(\log m)\right) .
$$

Здесь $(m-n) / m \geqslant \frac{1}{2}$,

$$
\begin{aligned}
& B^{-1}(\log n)=(\log n)^{-\alpha}(\log \log n)^{-\beta} \geqslant(\log m-1)^{-\alpha}(\log (\log m-1))^{-\beta}, \\
& B^{-1}(\log m)=(\log m)^{-\alpha}(\log \log m)^{-\beta} .
\end{aligned}
$$

Подставим в (18) и воспользуемся тем, что для любого $\eta>0$ при $m \geqslant m_{0}$ справедливо неравенство $\log \log m \leqslant D_{3}(\log m)^{\eta}$.

Лемма 10. Если $B(k)$ удовлетворяет условию (II) $п р и \alpha>0$, то при $k+p>k \geqslant k_{0}$ справедлива оченка

$$
\frac{1}{B(k)}-\frac{1}{B(k+p)} \geqslant C_{\alpha, \beta} \frac{p}{B(k)(k+p)} .
$$

Легко разбираются два случая - когда $B^{\prime}(k)$ убывает и когда $B(k)=k \varphi(k)$, где функция $\varphi(k)$ при $k \geqslant k_{0}$ возрастает.

Лемма 11. Пусть $B(k)$ удовлетворяет условию (II) $n р и \alpha>0$. Пусть $r \geqslant 2$ фиксировано, $3 \leqslant H<M / 8, M \geqslant 32$. Рассмотрим индексьь

$$
\left.m_{k}=m_{k}^{(r)}=\right] M 2^{r+k H M^{-1}}\left[, \quad k=0,1, \ldots, k_{1}, \quad k_{1}=\left[M(8 H)^{-1}\right] .\right.
$$

Тогда справедлива оченка при иельх $k<k+s \leqslant k_{1}$ :

$$
\rho_{k, s}^{2}=\left\|A_{m_{k}}-A_{m_{k+s}}\right\|^{2} \geqslant D_{0}^{2} \frac{s^{2} H^{2} \log (M /(4 s H))}{M^{2} B(r+\log (4 s H))(r+\log M)} .
$$

Д о к а за т ел ь с т в о. По лемме 1 , где $l_{k}=m_{k+s}-m_{k}$, имеем

$$
\rho_{k, s}^{2} \geqslant l_{k}^{2} m_{k+s}^{-2}\left(B^{-1}\left(\log l_{k}\right)-B^{-1}\left(\log m_{k+s}\right)\right) .
$$

Оценка этого выражения снизу аналогична оценке сверху в лемме 6 . Очевидно, что

$$
\begin{gathered}
l_{k} \geqslant m_{k+s}\left(1-2^{-s H M^{-1}}\right)-1 \geqslant s H(3 M)^{-1} m_{k+s}, \\
l_{k} m_{k+s}^{-1} \geqslant s H(3 M)^{-1}
\end{gathered}
$$

$\log l_{k} \leqslant r+\log (s H)+2, \quad \log M+r+2 \geqslant \log m_{k+s} \geqslant \log M+r$,

$$
M \geqslant 8 s H \text {. }
$$

Подставляя в выражение (20) и используя лемму 10, получим нужную оценку.

Теорема 6. Пусть для стационарной последовательности $\left(X_{k}\right)$ выполнень условия (I'), (II). Тогда энтропия множества средних $\left(A_{n}\right)$ допускает при $0<\varepsilon \leqslant \varepsilon_{0}$ нижние оченки, такие же по порядку, что и верхние оченки теорем 1-4. 
Например, при $\alpha=2, \beta=2$

$$
N(\varepsilon) \geqslant D_{2,2} \varepsilon^{-1} \log \log \log \varepsilon^{-1},
$$

а при $\alpha=2, \beta<2$

$$
N(\varepsilon) \geqslant D_{2, \beta} \varepsilon^{-1}\left(\log \log \varepsilon^{-1}\right)^{(2-\beta) / 2} .
$$

Д о к аз а т е л с с в о. Пусть фиксировано $M \geqslant M_{0}, \varepsilon=C_{0} / M$ (постоянные $C_{0}, M_{0}$ будут выбраны потом). Для оценки снизу $N(\varepsilon) \geqslant L_{M}$ по лемме 8 достаточно найти $L_{M} \varepsilon$-изолированных элементов последовательности $\left(A_{n}\right)$. Эти элементы будут иметь номера $m_{k}^{(r)}$, образованные согласно формуле (19), где $r_{M} \leqslant r \leqslant R_{M}, r$ - четные числа. Для разных значений $\alpha, \beta$ выбор $H(r), r_{M}, R_{M}$ будет различным. Рассмотрим сначала подробно случай $\alpha=2, \beta \leqslant 2$, а потом укажем необходимые изменения в доказательстве для остальных случаев.

В рассматриваемом случае положим

$$
H(r)=(B(r))^{1 / 2}=r(\log r)^{\beta / 2}, \quad r_{M}=2[\log \log M], \quad R_{M}=[\log M] .
$$

Упорядочим индексы $m_{k}^{(r)}$ из формулы (19) по возрастанию: $n_{1}<n_{2}<$ $\cdots<n_{L_{M}}$, при этом для числа $L_{M}$ получается оценка

$$
L_{M} \geqslant C M \sum r^{-1}(\log r)^{-\beta / 2}
$$

где сумма берется по четным числам $r$, удовлетворяющим неравенствам $2 \log \log M \leqslant r<[\log M]$. Поэтому $L_{M}$ при $\beta=2$ превосходит выражение $D M \log \log \log M$, а при $\beta<2$ - выражение $D M(\log \log M)^{(2-\beta) / 2}$. Покажем, что

$$
\left\|A_{n_{i}}-A_{n_{j}}\right\|>\frac{2 C_{0}}{M} \quad(i \neq j) .
$$

a) Пусть сначала $n_{i}, n_{j}$ из одной $r$-й серии,

$$
n_{i}=m_{k}^{(r)}=m_{k}, \quad n_{j}=m_{k+s}^{(r)}=m_{k+s}, \quad s \geqslant 1, \quad l_{k}=m_{k+s}-m_{k} .
$$

По лемме 11

$$
\rho_{k, s}^{2} \geqslant D_{0}^{2} A^{2} M^{-2}
$$

где

$$
A^{2}=A^{2}(r, s, M)=\frac{s^{2} H^{2}(r) \log (M /(4 s H))}{B(r+\log (4 s H)+2)(r+\log M)} .
$$

Оценим $A^{2}$ снизу. Если $M / 2 \geqslant 4 s H \geqslant \log ^{2} M$, то при $M \geqslant M_{0}$

$$
A^{2} \geqslant \frac{D_{2} \log ^{4} M}{B(2 \log M) \cdot 2 \log M} \geqslant D_{1}^{2},
$$

так как $B(2 \log M) \sim(2 \log M)^{2}(\log \log M)^{\beta} \leqslant C(\log M)^{5 / 2}$.

Если $4 s H<\log ^{2} M$, то

$$
\log \frac{M}{4 s H} \sim \log M, \quad \frac{B(r)}{B(r+2 \log \log M)} \geqslant \frac{1}{5}, \quad \frac{\log M}{r+\log M} \geqslant \frac{1}{2},
$$


и снова при $M \geqslant M_{0}$ имеем $A^{2} \geqslant D_{1}^{2}$. Итак, при $M \geqslant M_{0}$

$$
\rho_{k, s}^{2}>\frac{C_{1}^{2}}{M^{2}} \text {. }
$$

б) Пусть индексы $n_{i}, n_{j}$ из разных серий,

$$
n=n_{i}=m_{k_{1}}^{\left(r_{1}\right)}, \quad m=n_{j}=m_{k_{2}}^{\left(r_{2}\right)}, \quad r_{2} \geqslant r_{1}+2 .
$$

Тогда $m \geqslant 2 n$ и по лемме 9 , где можно взять $\delta=4>\alpha+1$, имеем

$$
\left\|A_{n_{i}}-A_{n_{j}}\right\|^{2} \geqslant \frac{D_{4}}{\log ^{4} m} \geqslant \frac{D_{3}}{\log ^{4} M}>\frac{C_{2}^{2}}{M^{2}} \quad\left(M \geqslant M_{0}\right),
$$

так как в конструкции (19) $m \leqslant 2 M^{2}$. Неравенства (23) и (24) означают $\varepsilon$-изолированность элементов $\left(n_{i}: 1 \leqslant i \leqslant L_{M}\right), \varepsilon=C_{0} / M, 2 C_{0}=$ $\min \left(C_{1}, C_{2}\right)$. По лемме 8 из $(21)$ следует теорема 6 при $\alpha=2, \beta \leqslant 2$.

Укажем небольшие изменения в доказательстве для остальных случаев.

Если $1<\alpha<2, \beta-$ любое, то берем в (19)

$$
H(r)=(B(r))^{1 / 2}, \quad r_{M}=[\log M], \quad R_{M}=2[\log M] .
$$

Если $\alpha=1, \beta-$ любое, то полагаем

$$
H(r)=(r B(r))^{1 / 2}(\log M)^{-1 / 2}, \quad r_{M}=[\log M], \quad R_{M}=[\sqrt{M}] .
$$

При $0<\alpha<1$ и любом $\beta$ проще всего рассмотреть множество средних

$$
\begin{gathered}
S=\left\{A_{2^{k}}: \frac{M_{1}}{2}<k<M_{1}\right\}, \quad M_{1}=M^{2 /(1+\alpha)}(\log M)^{-\beta /(1+\alpha)}, \\
\operatorname{Card} S \sim \frac{M_{1}}{2} .
\end{gathered}
$$

Тогда по лемме 1 при $M_{1} / 2<k<j<M_{1}$ имеем

$$
\begin{aligned}
\left\|A_{2^{k}}-A_{2^{j}}\right\|^{2} & \geqslant \frac{1}{4}\left(\frac{1}{k^{\alpha}(\log k)^{\beta}}-\frac{1}{(k+1)^{\alpha}(\log (k+1))^{\beta}}\right) \\
& \geqslant C_{\alpha, \beta} \frac{1}{k^{\alpha+1}(\log k)^{\beta}} \geqslant \frac{C_{2}^{2}}{M^{2}} .
\end{aligned}
$$

Наконец, при $\alpha=0, \beta>0$ образуем множество $S$ из элементов $A_{n}$ с номерами $m_{k}, k=1, \ldots, K_{M}$, где

$$
K_{M}=\left[\frac{M^{2}}{\log ^{\beta} M^{2}}\right], \quad m_{k}=\left[2^{n_{k}}\right], \quad n_{k}=2^{p_{k}}, \quad p_{k}=\left(\frac{M^{2}}{k}\right)^{1 / \beta},
$$

$$
\text { Card } S=K_{M} \text {. }
$$

Проводя такие же оценки, как в доказательстве леммы 56, получим, что для $1 \leqslant k<j \leqslant K_{M}$

$$
\left\|A_{m_{k}}-A_{m_{j}}\right\|^{2} \geqslant \frac{1}{2}\left[R\left(m_{k}\right)-R\left(m_{j}\right)\right] \geqslant \frac{C_{2}^{2}}{M^{2}} .
$$

Это заканчивает доказательство теоремы 6. 
3. Один класс стационарных последовательностей. В этом пункте построены примеры стационарных последовательностей, для которых реализуются оценки (I), (I'), (II) и, таким образом, справедливы точные по порядку оценки энтропии $N(\varepsilon)$.

Начнем со следующей леммы. Пусть $\left(X_{k}\right)$ - стационарная последовательность с корреляционной функцией $R_{1}(n)$, пусть функции $F_{0}(n), F_{1}(n), R(n)$ определены по формуле $(1)$ п. 1. В настоящем пункте удобнее использовать натуральные логарифмы, положим также

$$
L_{n}=\ln n \quad(n \geqslant 2), \quad L_{n}=1 \quad(n=1) .
$$

Лемма 12. Пусть при некоторых $A>0, \alpha>0$ и $B$ выполнено соотношение

$$
R_{1}(n)=A L_{n}^{-\alpha}+B L_{n}^{-\alpha-1}+O\left(L_{n}^{-\alpha-2}\right)
$$

Tozda

$$
\begin{aligned}
& F_{0}(n)=A n L_{n}^{-\alpha}+(A \alpha+B) n L_{n}^{-\alpha-1}+O\left(n L_{n}^{-\alpha-2}\right) \\
& F_{1}(n)=\frac{A n^{2}}{2} L_{n}^{-\alpha}+\left(\frac{A \alpha}{4}+\frac{B}{2}\right) n^{2} L_{n}^{-\alpha-1}+O\left(n^{2} L_{n}^{-\alpha-2}\right), \\
& R(n)=R_{1}(0) n^{-1}+A L_{n}^{-\alpha}+\left(\frac{3 A \alpha}{2}+B\right) L_{n}^{-\alpha-1}+O\left(L_{n}^{-\alpha-2}\right), \\
& R(n)-R(n+1)=A \alpha n^{-1} L_{n}^{-\alpha-1}\left(1+O\left(L_{n}^{-1}\right)\right) .
\end{aligned}
$$

Д о к а з а т е л ь с т в о. Формулы (26)-(28) получаются суммированием по частям в суммах

$$
\sum_{k=1}^{n} L_{k}^{-\alpha}, \quad \sum_{k=1}^{n} k L_{k}^{-\alpha}, \quad \sum_{k=1}^{n} L_{k}^{-\alpha-1} .
$$

Обозначим $\Delta f(n)=f(n+1)-f(n)$. Легко проверяется, что

$$
\Delta\left(n^{2} R(n)\right)=R_{1}(0)+2 F_{0}(n) .
$$

С другой стороны, это выражение равно $(n+1)^{2} \Delta R(n)+(2 n+1) R(n)$. Поэтому

$$
\Delta R(n)=2(n+1)^{-2}\left(F_{0}(n)-n R(n)\right)+O\left(n^{-2}\right),
$$

и формула (29) получается подстановкой формул (26) и (28).

Лемма 13. Если выполнено условие (25), то при $n_{0} \leqslant n<m$ справедливь неравенства

$$
D_{1}\left(L_{n}^{-\alpha}-L_{m}^{-\alpha}\right) \leqslant R(n)-R(m) \leqslant D_{2}\left(L_{n}^{-\alpha}-L_{m}^{-\alpha}\right) .
$$

Действительно, по лемме 12

$$
B_{1} n^{-1} L_{n}^{-\alpha-1} \leqslant R(n)-R(n+1) \leqslant B_{2} n^{-1} L_{n}^{-\alpha-1} \quad\left(n \geqslant n_{0}\right) .
$$


Кроме того,

$$
C_{1}\left(L_{n}^{-\alpha}-L_{n+1}^{-\alpha}\right) \leqslant n^{-1} L_{n}^{-\alpha-1} \leqslant C_{2}\left(L_{n}^{-\alpha}-L_{n+1}^{-\alpha}\right) \quad(n \geqslant 2) .
$$

Записывая $R(n)-R(m)=\sum_{k=n}^{m-1}(R(k)-R(k+1))$ и используя эти оценки, получим утверждение леммы 13.

Леммы 12 и 13 показывают, что если корреляционная функция стационарной последовательности $\left(X_{k}\right)$ удовлетворяет условию $(25)$, то выполнены предположения (I), (I'), (II) из пп. 1 и 2 при $B(u)=u^{\alpha}, \alpha>0$. Поэтому для системы $\left(X_{k}\right)$ справедливы как верхние, так и нижние оценки энтропии средних $N(\varepsilon)$, полученные выше.

Наша следующая цель - показать, что существует класс стационарных последовательностей, для которых предположение (25) действительно выполняется.

Рассмотрим стационарную последовательность $\left(X_{n}\right)$, образованную процессом скользяшего суммирования

$$
X_{n}=\sum_{k=1}^{\infty} c_{k} \Phi_{k+n}
$$

где $\left(\Phi_{n}\right)$ - произвольная ортонормированная система в $L^{2}(\Omega), \mathbf{E} \Phi_{n}=0$, $\left(c_{n}\right) \in l^{2}$. Корреляционная функция $R_{1}(n)$ имеет вид

$$
R_{1}(n)=\sum_{k=1}^{\infty} c_{k} c_{k+n}, \quad n=0,1,2, \ldots .
$$

Нас интересует асимптотика $R_{1}(n)$ в зависимости от скорости убывания коэффициентов $\left(c_{n}\right)$. Оценить порядок убывания $R_{1}(n)$ можно по следующей лемме. Пусть $f(k)$ - медленно меняющаяся положительная функция, т.е. $f(c k) / f(k) \rightarrow 1$ при $k \rightarrow \infty$ для всех $c>0$.

Лемма 14. Предположим, что

$$
c_{k}=(k f(k))^{-1 / 2}, \quad \sum c_{k}^{2}<\infty, \quad c_{k} \downarrow 0, \quad g(n)=\sum_{k=n+1}^{\infty} c_{k}^{2} .
$$

Tогда

$$
g(n) \leqslant R_{1}(n) \leqslant g(n)+C(f(n))^{-1} .
$$

Действительно, в силу монотонного убывания коэффициентов левое неравенство очевидно. Далее,

$$
R_{1}(n) \leqslant \sum_{k=n+1}^{\infty} c_{k}^{2}+c_{n+1} \sum_{k=1}^{n} c_{k}
$$

и для медленно меняюшейся функции $f(k)$ второе слагаемое есть $O\left((f(n))^{-1}\right)$. В частности, для главного интересующего нас случая, когда $f(1)=1, f(k)=(\ln k)^{2 \beta}(k \geqslant 2), \beta>\frac{1}{2}$, из $(31)$ получаем оценку

$$
R_{1}(n) \sim A_{\alpha}(\ln n)^{-\alpha}, \quad \alpha=2 \beta-1>0 .
$$

Однако нам нужна более точная асимптотика функции $R_{1}(n)$. 
Лемма 15. Если $\left(X_{k}\right)$ - стаиионарная последовательность, образованная по формуле (30), где $c_{k}=k^{-1 / 2} L_{k}^{-\beta}, \beta>\frac{1}{2}$, то для коррелячионной Функиии $R_{1}(n)$ справедлива формула (25), где $\alpha=2 \beta-1$, $A=\alpha^{-1}, B=\ln 4$.

Д о к а зате л ь с т о. При оценке выражения $R_{1}(n)$ заменим сумму $\sum_{k=1}^{\infty} c_{k} c_{k+n}$ интегралом

$$
J_{n}=\int_{2}^{\infty} \frac{d x}{\sqrt{x(x+n)}(\ln x)^{\beta}(\ln (x+n))^{\beta}},
$$

с погрешностью $O\left(n^{-1 / 2}\right)$, что заведомо меньше остаточного члена в формуле (25). Обозначим

$$
J_{n}=I_{n}+K_{n}, \quad I_{n}=\int_{2}^{n / 2}, \quad K_{n}=\int_{n / 2}^{\infty} .
$$

Покажем, что

$$
\begin{aligned}
I_{n} & =B_{1} L_{n}^{-2 \beta}+O\left(L_{n}^{-2 \beta-1}\right), \\
K_{n} & =\alpha^{-1} L_{n}^{-2 \beta+1}+B_{2} L_{n}^{-2 \beta}+O\left(L_{n}^{-2 \beta-1}\right),
\end{aligned}
$$

где $B_{1}+B_{2}=\ln 4$.

Оченка $I_{n}$. Представим $I_{n}=I_{n}^{\prime}+I_{n}^{\prime \prime}$, где $I_{n}^{\prime}$ - интеграл от 2 до $\sqrt{n}$, $I_{n}^{\prime \prime}$ - интеграл от $\sqrt{n}$ до $n / 2$. Очевидно, что $I_{n}^{\prime}=O\left(n^{-1 / 4}\right)$. Замена $x=n y$ дает:

$$
I_{n}^{\prime \prime}=L_{n}^{-2 \beta}\left(\int_{n^{-1 / 2}}^{1 / 2}(y(y+1))^{-1 / 2} d y+\int_{n^{-1 / 2}}^{1 / 2} G(y)(y(y+1))^{-1 / 2} d y\right) .
$$

Здесь

$G(y)=\left(1+L_{n}^{-1} \ln y\right)^{-\beta}\left(1+L_{n}^{-1} \ln (y+1)\right)^{-\beta}-1=O\left(L_{n}^{-1}(|\ln y|+|\ln (y+1)|)\right)$, так как при $n^{-1 / 2} \leqslant y \leqslant \frac{1}{2}$ имеем $L_{n}^{-1}|\ln y| \leqslant \frac{1}{2}, L_{n}^{-1}|\ln (y+1)| \leqslant \frac{1}{2}$. Далее,

$$
\int_{0}^{1 / 2}(y(y+1))^{-1 / 2} d y=\int_{1 / 2}^{1}\left(t^{2}-\frac{1}{4}\right)^{-1 / 2} d t=\ln (2+\sqrt{3}) .
$$

Собирая эти оценки, получим соотношение $(32)$, где $B_{1}=\ln (2+\sqrt{3})$.

Оченка $K_{n}$. Замены $x=n y, t=y+\frac{1}{2}$ дают

$$
K_{n}=\int_{1}^{\infty}\left(t^{2}-\frac{1}{4}\right)^{-1 / 2}\left(\ln \left(t-\frac{1}{2}\right)+L_{n}\right)^{-\beta}\left(\ln \left(t+\frac{1}{2}\right)+L_{n}\right)^{-\beta} d t .
$$

Для оценки скобок, содержащих логарифмы, представим их в виде $(\ln t+$ $\left.L_{n}\right)^{-2 \beta}\left(1+O\left(t^{-1} L_{n}^{-1}\right)\right)$.

Поэтому

$K_{n}=K_{n}^{\prime}+K_{n}^{\prime \prime}, \quad K_{n}^{\prime \prime}=O\left(L_{n}^{-2 \beta-1}\right), \quad K_{n}^{\prime}=\int_{1}^{\infty}\left(t^{2}-\frac{1}{4}\right)^{-1 / 2}\left(\ln t+L_{n}\right)^{-2 \beta} d t$. 
Обозначим

$$
\begin{gathered}
\Phi(t)=\ln \left(t+\sqrt{t^{2}-\frac{1}{4}}\right)-\ln (2 t), \quad \Phi(\infty)=0, \\
\Phi(1)=\ln (2+\sqrt{3})-\ln 4, \quad \Phi(t)=O\left(t^{-2}\right) \quad(t \rightarrow \infty) .
\end{gathered}
$$

Интегрируя, получаем

$$
\begin{aligned}
K_{n}^{\prime} & =\int_{1}^{\infty} t^{-1}\left(\ln t+L_{n}\right)^{-2 \beta} d t+\int_{1}^{\infty}\left(\ln t+L_{n}\right)^{-2 \beta} d \Phi(t) \\
& =\alpha^{-1} L_{n}^{-2 \beta+1}-\Phi(1) L_{n}^{-2 \beta}+O\left(L_{n}^{-2 \beta-1}\right),
\end{aligned}
$$

и для $K_{n}$ верна формула $(33)$, где $B_{2}=\ln 4-\ln (2+\sqrt{3})$.

Поэтому справедлива асимптотика (25) для $J_{n}=I_{n}+K_{n}$.

Применяя леммы $12-15$ и результаты пп. 1 и 2 , получаем следующую теорему.

Теорема 7. Рассмотрим стаиионарную последовательность, определенную формулой $(30)$, где $c_{1}=1, c_{k}=k^{-1 / 2}(\ln k)^{-\beta} \quad(k \geqslant 2)$, $\left(\Phi_{k}\right)$ - произвольная ортонормированная система, $\alpha=2 \beta-1>0$. Для энтропии множества средних $A_{n}$ этой последовательности справедливы точные по порядку оченки

$$
C_{\alpha} \Psi_{\alpha}(\varepsilon) \leqslant N(\varepsilon) \leqslant D_{\alpha} \Psi_{\alpha}(\varepsilon),
$$

где при $\alpha>2, \alpha=2,1<\alpha<2, \alpha=1,0<\alpha<1$ функиия $\Psi_{\alpha}(\varepsilon)$ равна соответственно

$$
\varepsilon^{-1}, \quad \varepsilon\left(\log \log \varepsilon^{-1}\right), \quad \varepsilon^{-1}\left(\log \varepsilon^{-1}\right)^{(2-\alpha) / 2}, \quad \varepsilon^{-1}\left(\log \varepsilon^{-1}\right)^{3 / 2}, \quad \varepsilon^{-2 /(\alpha+1)} .
$$

3 а м е ч а н и е 2. Нетрудно проверить, что правое неравенство леммы 13 справедливо при более общем предположении

$$
R_{1}(n)=A L_{n}^{-\alpha}\left(1+O\left(L_{n}^{-1}\right)\right) .
$$

Таким образом, уже при этом предположении верны верхние оценки энтропии средних $N(\varepsilon)$, полученные в п. 1 (при $\alpha>0, \beta=0$ ). Однако для двусторонних оценок энтропии приходится использовать более точную асимптотику корреляционной функции.

3 а м е ч а н и е 3 . Мы ограничились здесь «чисто логарифмической» шкалой для $R_{1}(n)$ и $R(n)$. Аналогичные результаты можно получить и в шкале с повторными логарифмами, взяв

$$
c_{k}=k^{-1 / 2}(\ln k)^{-\beta}(\ln \ln k)^{-\gamma}, \quad k \geqslant 3 .
$$

3 а м е ч а н и е 4. Как известно, спектральная плотность $f(\lambda)$ последовательности $\left(X_{k}\right)$ имеет вид

$$
f(\lambda)=\left|\sum_{k=1}^{\infty} c_{k} e^{i k \lambda}\right|^{2}=\left(\sum_{k=1}^{\infty} c_{k} \cos k \lambda\right)^{2}+\left(\sum_{k=1}^{\infty} c_{k} \sin k \lambda\right)^{2}
$$


Для рассматриваемого случая, когда

$$
c_{k}=k^{-1 / 2}(\ln k)^{-(\alpha+1) / 2}, \quad \alpha>0, \quad k \geqslant 2,
$$

известные результаты по асимптотике тригонометрических рядов (см. [6, с. 300]) дают следующую оценку:

$$
f(\lambda) \leqslant C_{1}|\lambda|^{-1}\left(\ln \frac{2 \pi}{|\lambda|}\right)^{-(\alpha+1)}, \quad 0<|\lambda| \leqslant \pi .
$$

В заключение получим оценки энтропии $N(\varepsilon)$ при ограничениях на спектральную плотность последовательности $\left(X_{k}\right)$ типа условия $(34)$. Известна следующая связь функции $R(n)$ и спектральной плотности:

$$
R(n)=\int_{-\pi}^{\pi} K_{n}(\lambda) f(\lambda) d \lambda, \quad K_{n}(\lambda)=\frac{\sin ^{2} n \lambda / 2}{\pi n^{2} \sin ^{2} \lambda / 2}
$$

Поэтому скорость убывания $R(n)$ связана с ограничениями на поведение спектральной плотности при $\lambda \rightarrow 0$. Подробнее об этой связи см., например, работы [7]-[9].

Будем говорить, что стационарная последовательность принадлежит классу $S_{\gamma, \delta}$, если она имеет спектральную плотность, удовлетворяющую условию

$$
f(\lambda) \leqslant C|\lambda|^{-1}\left(\ln \frac{2 \pi}{|\lambda|}\right)^{-\gamma}\left(\ln \ln \frac{4 \pi}{|\lambda|}\right)^{-\delta} \quad(0<|\lambda| \leqslant \pi) .
$$

Так как $f(\lambda) \in L(\Omega)$, то естественно считать, что либо $\gamma>1, \delta$ любое, либо $\gamma=1, \delta>1$. Обозначим в первом случае $\alpha=\gamma-1, \beta=\delta$, а во втором $\alpha=0, \beta=\delta-1$.

Лемма 16. $Е с л и ~\left(X_{k}\right) \in S_{\gamma, \delta}$, то выполнень условия (I), (II) $n .1$, m.e. $n p u 2<n<m$

$$
|R(n)-R(m)| \leqslant D_{1}\left((\ln n)^{-\alpha}(\ln \ln n)^{-\beta}-(\ln m)^{-\alpha}(\ln \ln m)^{-\beta}\right) .
$$

Обозначим $L_{n}(\lambda)=K_{n}(\lambda)-K_{n+1}(\lambda)$. Для доказательства леммы 16 нужны следующие оценки ядер $L_{n}(\lambda)$.

Лемма 17. Справедливь следующие неравенства: $\left|L_{n}(\lambda)\right| \leqslant$ $C_{1} n \lambda^{2} n p u|\lambda| n \leqslant 1,\left|L_{n}(\lambda)\right| \leqslant C_{2} n^{-2}|\lambda|^{-1} n p u|\lambda| n>1$.

Д о к а з а т е л с с т о. Имеем:

$$
\begin{aligned}
\left|L_{n}(\lambda)\right| & \leqslant C \lambda^{-2}\left|M_{n}(\lambda)\right|, \\
M_{n}(\lambda) & =n^{-2} \sin ^{2} \frac{n \lambda}{2}-(n+1)^{-2} \sin ^{2} \frac{(n+1) \lambda}{2} .
\end{aligned}
$$

Для оценки $M_{n}(\lambda)$ применим формулу Лагранжа:

$$
M_{n}(\lambda)=\left.\frac{\partial}{\partial u}\left(u^{-2} \sin ^{2} \frac{u \lambda}{2}\right)\right|_{u=n+\theta}, \quad 0<\theta<1 .
$$


Поэтому $\left|M_{n}(\lambda)\right|$ равен значению при $u=n+\theta$ функции

$$
\frac{|u \lambda \sin u \lambda-2(1-\cos u \lambda)|}{2 u^{3}} \text {. }
$$

Легко проверяется, что при $u|\lambda| \leqslant 1$ числитель есть $O\left((u \lambda)^{4}\right)$, при $u|\lambda|>1$ он не превосходит $5 u|\lambda|$. Из (36), (37) получаем нужные оценки.

Доказательст в о л е м м ы 16 . Оценим сначала разность $\Delta R(n)=R(n+1)-R(n)$. Для $f(\lambda) \in S_{\gamma, \delta}$ имеем:

$$
\begin{aligned}
|\Delta R(n)| \leqslant & \int_{-\pi}^{\pi} f(\lambda)\left|L_{n}(\lambda)\right| d \lambda \leqslant C_{1} \int_{n|\lambda|<1} n|\lambda|\left(\ln \frac{2 \pi}{|\lambda|}\right)^{-\gamma}\left(\ln \ln \frac{4 \pi}{|\lambda|}\right)^{-\delta} d \lambda \\
& +C_{2} \int_{n|\lambda|>1} n^{-2} \lambda^{-2}\left(\ln \frac{2 \pi}{|\lambda|}\right)^{-\gamma}\left(\ln \ln \frac{4 \pi}{|\lambda|}\right)^{-\delta} d \lambda \\
= & O\left(n^{-1}(\ln n)^{-\gamma}(\ln \ln n)^{-\delta}\right) .
\end{aligned}
$$

Так как $|R(n)-R(m)| \leqslant \sum_{k=m}^{n}|\Delta R(k)|$, то из (38) вытекает утверждение леммы.

Поэтому к стационарным последовательностям класса $S_{\gamma, \delta}$ применимы верхние оценки энтропии из теорем 1-4. Ограничимся случаем $\gamma=\alpha+1, \alpha>0, \beta=0$, будем обозначать $S_{\alpha+1,0}=S_{\alpha+1}$.

Теорема 8. а) Если $\left(X_{k}\right) \in S_{\alpha+1}$, то справедливь оченки

$$
N(\varepsilon) \leqslant C_{\alpha} \Psi_{\alpha}(\varepsilon),
$$

где функиия $\Psi_{\alpha}(\varepsilon)$ та же самая, что в теореме 7.

б) Для любого $\alpha>0$ существует стаиионарная последовательность $\left(X_{k}\right) \in S_{\alpha+1}$ maкая, ито

$$
N(\varepsilon) \geqslant D_{\alpha} \Psi_{\alpha}(\varepsilon)
$$

Д о к а з а т е л с с в о. Первая часть теоремы - переформулировка теорем $1-4$ в случае $\alpha=\gamma-1, \beta=0$ (с использованием леммы 16). Далее, в силу замечания 2 , стационарная последовательность $\left(X_{k}\right)$, образованная по формуле (30), где

$$
c_{k}=k^{-1 / 2}(\ln k)^{-(\alpha+1) / 2} \quad(k \geqslant 2), \quad \alpha>0,
$$

имеет спектральную плотность класса $S_{\alpha+1}$. В то же время по теореме 7 для нее справедливы нижние оценки (39).

3 а м е ч а н и е 5 . Это утверждение дает более точные оценки энтропии для $\left(X_{k}\right) \in S_{\alpha+1}$, чем теорема из работы [3]. Действительно, для $\left(X_{k}\right) \in S_{\alpha+1}$ выполнено соотношение

$$
\int_{|\lambda| \leqslant \lambda_{0}}\left(\log \lambda^{-1}\right)^{\eta} f(\lambda) d \lambda<\infty
$$


при $\eta<\alpha$, т.е. по теореме из [3] получалась бы оценка

$$
N(\varepsilon)=O\left(\varepsilon^{-2}\left(\log \varepsilon^{-1}\right)^{-\eta}\right)
$$

Порядок этой оценки для всех $\alpha$ сильно отличается от порядка функции $\Psi_{\alpha}(\varepsilon)$ при $\varepsilon \rightarrow 0$.

\section{СПИСОК ЛИТЕРАТУРЫ}

1. Talagrand M. Applying a theorem of Fernique. - Ann. Inst. H. Poincaré, 1996, v. 32, №6, p. 779-799.

2. Weber $M$. Entropie métrique et convergence presque partout. Paris: Hermann, 1998, $151 \mathrm{p}$.

3. Gamet C., Weber $M$. Entropy numbers of some ergodic averages. - Теория вероятн. и ее примен., 1999 , т. 44 , в. 4, с. $776-795$.

4. Гапошкин В.Ф. Оценки энтропии множества средних некоторых классов стационарных и квазистационарных последовательностей. - Матем. заметки, 2005, т. 78 , № 1 , c. $52-58$.

5. Гапошкин $B . \Phi$. Точные оценки метрической энтропии множества средних квазистационарньх процессов. - Теория вероятн. и ее примен., 2006, т. 51, в. 4, c. $785-793$.

6. Зигмунд А. Тригонометрические ряды, т. І. М.: ИЛ, 1965, 615 с.

7. Гапошкин $B . \Phi$. Оценки средних для почти всех реализаций стационарных процессов. - Сиб. матем. журн., 1979, т. 20, № 5, с. 978-989.

8. Качуровский А. Г. Скорости сходимости в эргодических теоремах. - Успехи матем. наук, 1996, т. 51, № 4, с. 73-124.

9. Гапошкин В.Ф. О скорости убывания вероятностей $\varepsilon$-уклонений средних стационарных процессов. - Матем. заметки, 1998, т. 64, № 3, с. 366-372.

Поступила в редакцию 22.XII. 2005

Исправленный вариант

31.I. 2007 